International Mathematical Forum, Vol. 8, 2013, no. 3, 145 - 152

\title{
Some New Identities for Continued Fraction of Order Six
}

\author{
B. N. Dharmendra*, M. R. Rajesh Kanna*, R. Jagadeesh** \\ and S. Vasanth Kumar*** \\ *Post Graduate Department of Mathematics \\ Maharani's Science College for Women \\ J. L. B. Road,Mysore-570 001, India \\ bndharma@gmail.com, mr.rajeshkanna@gmail.com \\ **Department of Mathematics, Government Science College \\ N. T. Road, Bangalore-560 001, India \\ Jagadeeshr1978@gmail.com
***Department of Mathematics, The National Institute of Engineering Manandavadi Road, Mysore-570 008, India svkmaths.174@gmail.com

\begin{abstract}
In this paper, we establish some new modular relations between a continued fraction of order six $U(q)$ and $U\left(q^{n}\right)$ for $\mathrm{n}=6,10,14,22$.
\end{abstract}

Mathematics Subject Classification: Primary 11A55, 33D10

Keywords: Continued fraction, Theta functions

\section{INTRODUCTION}

In Chapter 16 of his second notebook [2], Ramanujan develops the theory of theta-function and is defined by

$$
\begin{aligned}
f(a, b) & :=\sum_{n=-\infty}^{\infty} a^{\frac{n(n+1)}{2}} b^{\frac{n(n-1)}{2}},|a b|<1, \\
& =(-a ; a b)_{\infty}(-b ; a b)_{\infty}(a b ; a b)_{\infty}
\end{aligned}
$$

where $(a ; q)_{0}=1$ and $(a ; q)_{\infty}=(1-a)(1-a q)\left(1-a q^{2}\right) \cdots$.

Following Ramanujan, we defined

$$
\varphi(q):=f(q, q)=\sum_{n=-\infty}^{\infty} q^{n^{2}}=\frac{(-q ;-q)_{\infty}}{(q ;-q)_{\infty}},
$$




$$
\begin{gathered}
\psi(q):=f\left(q, q^{3}\right)=\sum_{n=0}^{\infty} q^{\frac{n(n+1)}{2}}=\frac{\left(q^{2} ; q^{2}\right)_{\infty}}{\left(q ; q^{2}\right)_{\infty}}, \\
f(-q):=f\left(-q,-q^{2}\right)=\sum_{n=-\infty}^{\infty}(-1)^{n} q^{\frac{n(3 n-1)}{2}}=(q ; q)_{\infty}
\end{gathered}
$$

and

$$
\chi(q):=\left(-q ; q^{2}\right)_{\infty}
$$

The ordinary hypergeometric series ${ }_{2} F_{1}(a, b ; c ; x)$ is defined by

$$
{ }_{2} F_{1}(a, b ; c ; x)=\sum_{n=0}^{\infty} \frac{(a)_{n}(b)_{n}}{(c)_{n} n !} x^{n}
$$

where

$$
(a)_{0}=1,(a)_{n}=a(a+1)(a+2) \ldots(a+n-1), \text { for } n \geq 1,|x|<1 .
$$

Let

$$
z(r):=z(r ; x):={ }_{2} F_{1}\left(\frac{1}{r}, \frac{r-1}{r} ; 1 ; x\right)
$$

and

$$
q_{r}:=q_{r}(x):=\exp \left(-\pi c s c\left(\frac{\pi}{r}\right) \frac{{ }_{2} F_{1}\left(\frac{1}{r}, \frac{r-1}{r} ; 1 ; 1-x\right)}{{ }_{2} F_{1}\left(\frac{1}{r}, \frac{r-1}{r} ; 1 ; x\right)}\right) .
$$

where $r=2,3,4,6$ and $0<x<1$.

Let $n$ denote a fixed natural number, and assume that

$$
n \frac{{ }_{2} F_{1}\left(\frac{1}{r}, \frac{r-1}{r} ; 1 ; 1-\alpha\right)}{{ }_{2} F_{1}\left(\frac{1}{r}, \frac{r-1}{r} ; 1 ; \alpha\right)}=\frac{{ }_{2} F_{1}\left(\frac{1}{r}, \frac{r-1}{r} ; 1 ; 1-\beta\right)}{{ }_{2} F_{1}\left(\frac{1}{r}, \frac{r-1}{r} ; 1 ; \beta\right)},
$$

where $r=2,3,4$ or 6 . Then a modular equation of degree $n$ in the theory of elliptic functions of signature $r$ is a relation between $\alpha$ and $\beta$ induced by (1.6). We often say that $\beta$ is of degree $n$ over $\alpha$ and $m(r):=\frac{z(r: \alpha)}{z(r: \beta)}$ is called the multiplier. We also use the notations $z_{1}:=z_{1}(r)=z(r: \alpha)$ and $z_{n}:=z_{n}(r)=$ $z(r: \beta)$ to indicate that $\beta$ has degree $n$ over $\alpha$. When the context is clear, we omit the argument $r$ in $q_{r}, z(r)$ and $m(r)$.

The class invariant $G_{n}$ is defined by

$$
G_{n}:=2^{-\frac{1}{4}} q^{-\frac{1}{24}} \chi(q)=(4 \alpha(1-\alpha))^{-\frac{1}{24}} .
$$

The celebrated Rogers-Ramanujan continued fraction is defined as

$$
R(q):=\frac{q^{1 / 5} f\left(-q,-q^{4}\right)}{f\left(-q^{2},-q^{3}\right)}=\frac{q^{1 / 5}}{1}+\frac{q}{1}+\frac{q^{2}}{1}+\frac{q^{3}}{1}+\cdots,|q|<1,
$$

On page 365 of his Lost Notebook [8], Ramanujan recorded five identities showing the relationships between $R(q)$ and five continued fractions $R(-q), R\left(q^{2}\right)$, $R\left(q^{3}\right), R\left(q^{4}\right)$, and $R\left(q^{5}\right)$. He also recorded these identities at the scattered 
places of his Notebooks [7]. L. J. Rogers [9] established the modular equations relating $R(q)$ and $R\left(q^{n}\right)$ for $\mathrm{n}=2,3,5$, and 11 . The last of these equations cannot be found in Ramanujan's works.

The Ramanujan's cubic continued fraction $G(q)$ is defined as

$$
G(q):=\frac{q^{1 / 3} f\left(-q,-q^{5}\right)}{f\left(-q^{3},-q^{3}\right)}=\frac{q^{1 / 3}}{1}+\frac{q+q^{2}}{1}+\frac{q^{2}+q^{4}}{1}+\cdots,|q|<1,
$$

The continued fraction (1.9) was first introduced by Ramanujan in his second letter to G. H. Hardy [6]. He also recorded the continued fraction (1.9) on page 365 of his Lost Notebook [8] and claimed that there are many results for $G(q)$ similar the results obtained for the famous Rogers-Ramanujan continued fraction (1.8).

The Ramanjuan Göllnitz-Gordon continued fraction [6, p. 44], [4], [8] is defined as follows:

$$
H(q):=\frac{q^{1 / 2} f\left(-q^{3},-q^{5}\right)}{f\left(-q,-q^{7}\right)}=\frac{q^{1 / 2}}{1}+\frac{q^{2}}{1+q^{3}}+\frac{q^{4}}{1+q^{5}}+\cdots, \quad|q|<1,
$$

Motivated by the above cited works on the continued fractions, in this paper, we established the modular relation between continued fraction $U(q)$ and $U\left(q^{n}\right)$ for $n=6,10,14,22$.

$$
\begin{aligned}
& U(q):=q^{1 / 4} \frac{\left(q, q^{6}\right)\left(q^{5}, q^{6}\right)}{\left(q^{2}, q^{6}\right)\left(q^{4}, q^{6}\right)} \\
& =\frac{q^{-1 / 4}\left(1-q^{2}\right)}{\left(1-q^{3 / 2}\right)+} \frac{\left(1-q^{1 / 2}\right)\left(1-q^{7 / 2}\right)}{q^{1 / 2}\left(1-q^{3 / 2}\right)\left(1+q^{3}\right)+} \frac{\left(1-q^{5 / 2}\right)\left(1-q^{13 / 2}\right)}{q^{3 / 2}\left(1-q^{3 / 2}\right)\left(1+q^{6}\right)+\ldots}
\end{aligned}
$$

The continued fraction (1.11) was first established by K. R. Vasuki, N. Bhaskar and G. Sharath [10] $V(q)$ and $V\left(q^{n}\right)$ for $\mathrm{n}=2,3,5,7$ and 11.

\section{Preliminary Results}

In this section, we collect the necessary results required to prove our main results.

Lemma 2.1. [10] If $x:=U(q)$ and $y:=U\left(q^{2}\right)$, then

$$
x^{4}+3 x^{4} y^{2}-y^{2}+y^{4}=0 .
$$

Lemma 2.2. [10] If $x:=U(q)$ and $y:=U\left(q^{3}\right)$, then

$$
x^{3}-3 y^{3} x^{2}+3 y^{2} x-y=0 .
$$

Lemma 2.3. [10] If $x:=U(q)$ and $y:=U\left(q^{5}\right)$, then

$$
y^{6}+9 x^{5} y^{5}-5 x y^{5}+5 x^{2} y^{4}-5 x^{4} y^{2}+x y-5 x^{5} y-x^{6}=0 .
$$

Lemma 2.4. [10] If $x:=U(q)$ and $y:=U\left(q^{7}\right)$, then

$$
\begin{aligned}
& 21 x^{5} y^{5}-21 x^{7} y^{3}-21 x^{3} y^{7}+x y-7 x y^{5}+14 x^{2} y^{6} \\
& -7 x^{5} y-14 x^{6} y^{2}+y^{8}-x^{8}+27 x^{7} y^{7}+7 x^{3} y^{3}=0 .
\end{aligned}
$$


Lemma 2.5. [10] If $x:=U(q)$ and $y:=U\left(q^{11}\right)$, then

$$
\begin{aligned}
& y^{12}+x^{12}-495 x^{3} y^{7}+297 x^{5} y-495 x^{7} y^{3}+297 x^{3} y^{3} \\
& -308 x^{7} y^{7}-x^{11} y^{11}-22 x^{3} y^{11}+66 y^{10} x^{2}-243 x y \\
& -924 x^{5} y^{5}+297 x y^{5}+11 x^{11} y^{7}+92 x^{6} y^{6}-66 x^{9} y \\
& -165 x^{5} y^{9}+495 x^{8} y^{4}+495 x^{4} y^{8}+11 x^{7} y^{11}-66 x y^{9} \\
& +11 x^{9} y^{9}-165 x^{9} y^{5}-22 x^{11} y^{3}+66 x^{10} y^{2}=0 .
\end{aligned}
$$

\section{Relation Between $U(q)$ and $U\left(q^{n}\right)$}

Theorem 3.1. If $u:=U(q)$ and $v:=U\left(q^{6}\right)$, then

$$
\begin{aligned}
& \left(-9 v^{2}-27 v^{6}-1-27 v^{4}\right) u^{12}+\left(30 v^{2}+27 v^{8}+63 v^{6}\right. \\
& \left.+42 v^{4}-81 v^{10}-81 v^{12}\right) u^{8}+\left(-27 v^{6}-12 v^{2}-51 v^{4}\right. \\
& \left.+27 v^{10}+63 v^{8}\right) u^{4}+v^{2}+5 v^{4}+3 v^{6}-9 v^{8}=0 .
\end{aligned}
$$

Proof. Using the equations (2.1), then we have to express $y$ in terms of $x$.

$$
y=\sqrt{\frac{1}{2}-\frac{3}{2} x^{4}-\frac{1}{2} m} .
$$

where $m=\sqrt{9 x^{8}-10 x^{4}+1}$

Replace $q$ to $q^{2}$ in the equation (2.2) and using the above equation (3.2), we arrive at the equation (3.1).

Theorem 3.2. If $u:=U(q)$ and $v:=U\left(q^{10}\right)$, then

$$
\begin{aligned}
& \left(18 v^{2}+1+1458 v^{10}+135 v^{4}+729 v^{12}+540 v^{6}+1215 v^{8}\right) u^{24} \\
& +\left(-30 v^{4}+39366 v^{20}+1845 v^{6}+19683 v^{22}+10935 v^{18}-29160 v^{16}\right. \\
& \left.-2916 v^{10}-29160 v^{14}-10368 v^{12}-195 v^{2}\right) u^{20}+\left(300 v^{2}+4860 v^{18}\right. \\
& +169695 v^{16}-180 v^{10}-116640 v^{20}-43740 v^{22}+14385 v^{8}-945 v^{4} \\
& \left.-3030 v^{6}-66015 v^{12}+41310 v^{14}\right) u^{16}+\left(-24030 v^{18}+890 v^{6}+3840 v^{10}\right. \\
& +85860 v^{20}+1060 v^{4}-17160 v^{8}+31590 v^{22}-154440 v^{16}-130 v^{2} \\
& \left.-11520 v^{14}+84040 v^{12}\right) u^{12}+\left(-20 v^{6}-1530 v^{10}-7335 v^{12}-8100 v^{22}\right. \\
& \left.-160 v^{4}+9090 v^{18}+20 v^{2}-8505 v^{20}+60 v^{14}+14385 v^{16}+2095 v^{8}\right) u^{8} \\
& +\left(-30 v^{20}-40 v^{8}+108 v^{14}-5 v^{6}+585 v^{22}+120 v^{10}-128 v^{12}-615 v^{18}\right. \\
& \left.-v^{2}+6 v^{4}\right) u^{4}+v^{24}-20 v^{18}-6 v^{14}+15 v^{20}-6 v^{22}+15 v^{16}+v^{12}=0 .
\end{aligned}
$$

Proof. Using the equations (3.2) and replace $q$ to $q^{2}$ in the equation (2.3), we arrive at the equation (3.3). 
Theorem 3.3. If $u:=U(q)$ and $v:=U\left(q^{14}\right)$, then

$$
\begin{aligned}
& \left(-13608 v^{10}-1512 v^{6}-24 v^{2}-1-20412 v^{12}-6561 v^{16}-252 v^{4}-5670 v^{8}\right. \\
& \left.-17496 v^{14}\right) u^{32}+\left(101304 v^{8}+980 v^{2}-857304 v^{18}-4251528 v^{28}\right. \\
& -2480058 v^{26}+2204496 v^{20}+3306744 v^{24}+5373459 v^{22}-7616 v^{4} \\
& -540189 v^{14}+106974 v^{10}-104328 v^{12}-1594323 v^{30}-1248048 v^{16} \\
& \left.-10563 v^{6}\right) u^{28}+\left(-3024 v^{2}-40987296 v^{18}-2171736 v^{10}-37343754 v^{20}\right. \\
& +22228668 v^{24}-12124728 v^{26}+118244 v^{6}-3011526 v^{12}+7154 v^{4} \\
& +33985980 v^{22}+16226028 v^{14}+826686 v^{28}+17186904 v^{16}+4960116 v^{30} \\
& \left.+102284 v^{8}\right) u^{24}+\left(5866000 v^{12}-542136 v^{8}-5786802 v^{30}+13608 v^{4}\right. \\
& +60505704 v^{20}-24807888 v^{16}+3495975 v^{10}+3213 v^{2}+75997467 v^{18} \\
& -173824 v^{6}-76007484 v^{22}+29714769 v^{26}-54255096 v^{24}+13226976 v^{28} \\
& \left.-27250482 v^{14}\right) u^{20}+\left(-3433836 v^{12}-1718948 v^{10}+33483618 v^{24}\right. \\
& +3123036 v^{30}-30904524 v^{20}-1428 v^{2}-10961244 v^{28}+11408684 v^{16} \\
& +46411596 v^{22}-42649068 v^{18}+80052 v^{6}+14216356 v^{14}+413378 v^{8} \\
& \left.-15036 v^{4}-19452636 v^{26}\right) u^{16}+\left(-2814721 v^{14}+9083494 v^{18}+746984 v^{12}\right. \\
& -4879224 v^{24}+312788 v^{10}-13587 v^{6}+4693248 v^{26}+5866000 v^{20} \\
& +1102248 v^{28}+2016 v^{4}+294 v^{2}-780759 v^{30}-74424 v^{8}-10487925 v^{22} \\
& \left.-2756432 v^{16}\right) u^{12}+\left(616 v^{6}-15540 v^{10}-28 v^{2}+723912 v^{22}+14 v^{4}\right. \\
& +3388 v^{8}+168672 v^{14}+64386 v^{28}+212184 v^{16}+81648 v^{30}+102284 v^{24} \\
& \left.-354732 v^{26}-334614 v^{20}-51226 v^{12}-600964 v^{18}\right) u^{8}+\left(-8 v^{4}+392 v^{14}\right. \\
& -273 v^{10}+v^{2}-1288 v^{20}+3521 v^{26}-7616 v^{28}-3962 v^{22}-1712 v^{16} \\
& \left.+11256 v^{24}-2940 v^{30}+336 v^{12}+2223 v^{18}+56 v^{8}+14 v^{6}\right) u^{4}-28 v^{20} \\
& -v^{16}+56 v^{22}+8 v^{18}-28 v^{28}+56 v^{26}-70 v^{24}-v^{32}+8 v^{30}=0 . \\
& +12
\end{aligned}
$$

Proof. Using the equations (3.2) and replace $q$ to $q^{2}$ in the equation (2.4), we arrive at the equation (3.4).

Theorem 3.4. If $u:=U(q)$ and $v:=U\left(q^{22}\right)$, then

$$
\begin{aligned}
& \left(986040816 v^{40}+866052 v^{46}+5787668202778 v^{20}+172185640 v^{42}\right. \\
& +169882124472 v^{34}-25567718 v^{44}-9293998296 v^{38}-3107697009288 v^{6} \\
& +36295472877496 v^{18}+2121602538968 v^{16}+7169816404080 v^{26} \\
& -8124260320 v^{36}-1484627578016 v^{30}-4645309792068 v^{24}-67057229360 v^{32}
\end{aligned}
$$




$$
\begin{aligned}
& +7526562463596 v^{8}+1153816174106 v^{28}-33822367464468 v^{14} \\
& -21407327495664 v^{22}+16249149212472 v^{10}-90004495680 v^{2} \\
& \left.-10144434386148 v^{12}-1689359173194 v^{4}\right) u^{40}+\left(-3273211848740 v^{24}\right. \\
& +738329332176 v^{14}-40302183174 v^{34}-10034825676276 v^{20} \\
& -13900658361876 v^{12}+878301864495 v^{6}-101694712680 v^{4}-3740 v^{46} \\
& +2954425676 v^{36}-11067373615958 v^{22}+236555666724 v^{30}+320144 v^{44} \\
& +1034347215 v^{38}-20009092 v^{42}+1049419250820 v^{8}+13667984126888 v^{18} \\
& +959647496676 v^{26}+1784257035924 v^{28}-199694118756 v^{32} \\
& \left.+24665123615220 v^{16}+52161040 v^{40}-5381586440208 v^{10}+49220622 v^{2}\right) u^{12} \\
& +\left(-715417510156 v^{24}-33360756984 v^{34}-3865351731692 v^{16}-177147 v^{46}\right. \\
& +4000764020 v^{36}+3875886471700 v^{22}-1939322249040 v^{8}+190669714684 v^{6} \\
& +4596069155997 v^{14}-32563650724 v^{32}+375181768896 v^{4}+3811754547520 v^{12} \\
& +2179277770780 v^{20}-1440521010324 v^{26}+4958612 v^{44}-242137108 v^{40} \\
& +298590324054 v^{30}-32418804 v^{42}-5774844512356 v^{18}-1743267899032 v^{10} \\
& \left.+170319058652 v^{28}+1801417464 v^{38}+40572575868 v^{2}\right) u^{44}+\left(-672672 v^{46}\right. \\
& +1259642544930 v^{4}-213474556752 v^{34}-9163994670848 v^{24}-257049452 v^{42} \\
& -154715374417744 v^{18}+27681311067504 v^{14}+114690778770656 v^{20} \\
& +610216920792 v^{8}+330768452168 v^{32}+137744426798440 v^{22}-60498408 v^{40} \\
& +92105320977390 v^{12}-30187877125560 v^{26}-194969404867512 v^{16} \\
& +3021711821168 v^{30}-17484707970 v^{36}+10551429891684 v^{6}+25208370 v^{44} \\
& +12279205620 v^{38}-1703798470176 v^{28}-15520421664 v^{2} \\
& \left.+5496853742820 v^{10}\right) u^{24}+\left(-1153986564 v^{40}+1215992658672 v^{4}+459756 v^{46}\right. \\
& -2697448160 v^{38}-3520165727716 v^{26}-18738939663123 v^{8}+182245316 v^{42} \\
& +77704695629262 v^{12}+822524400988 v^{30}+69665179936 v^{36}-15497108 v^{44} \\
& +10478952028842 v^{28}-1225146773983 v^{32}+30959507291192 v^{18} \\
& -50016236089561 v^{24}-141321547999313 v^{16}+47705515968792 v^{10} \\
& -66064283852688 v^{14}+2264361946264 v^{22}+120301867133228 v^{20} \\
& \left.-9479924411760 v^{6}-88508190672 v^{2}-41371403616 v^{34}\right) u^{32}+\left(3487088 v^{44}\right. \\
& +159366000917640 v^{16}-2239286841198 v^{6}+35971419 v^{42}-723099316 v^{40} \\
& -361512569928 v^{30}-23820883717257 v^{10}-103497180951340 v^{22}-114356 v^{46} \\
& -88146271283136 v^{20}-631047903112 v^{32}+3536210601 v^{2}+554962826 v^{38}
\end{aligned}
$$




$$
\begin{aligned}
& -13174667920764 v^{8}-1216990926544 v^{24}-62092692238104 v^{12} \\
& +1166586125364 v^{4}+4090465135592 v^{28}-2599813287924 v^{14} \\
& +16879599634590 v^{26}+116117584643866 v^{18}+29466128204 v^{36} \\
& \left.-42618798387 v^{34}\right) u^{20}+\left(3095081649 v^{16}-108948114 v^{20}-14271253308 v^{12}\right. \\
& -7766502216 v^{10}-399142932 v^{18}-6018313554 v^{4}-531441 v^{24}+14875836 v^{22} \\
& \left.-1+766634616 v^{14}+18641257569 v^{8}+13214331852 v^{6}-7872923556 v^{2}\right) u^{48} \\
& +\left(+43706520 v^{44}-14346731102964 v^{12}+77402091902322 v^{14}-1395306 v^{46}\right. \\
& -44249452367285 v^{10}+33337235568692 v^{22}-55314629077356 v^{20} \\
& +1909836815336 v^{30}+28409245036860 v^{24}+15071777424 v^{38} \\
& -843039580 v^{40}-325887925 v^{42}+112874578740 v^{2}+692025090900 v^{32} \\
& +48686814300476 v^{16}-68629953513708 v^{18}-6639961932596 v^{28} \\
& +2047048773264 v^{4}-239874223632 v^{34}-3699702295920 v^{8} \\
& \left.-22233126036 v^{36}-9475829967054 v^{26}+9963537817676 v^{6}\right) u^{36} \\
& +\left(154696957 v^{40}-48884627296404 v^{18}-3528733232948 v^{28}+174064 v^{46}\right. \\
& +43951142923578 v^{20}-12308846722 v^{36}-5960592 v^{44}+437056008367 v^{32} \\
& +105771886704 v^{34}+42663462395532 v^{22}+3572185950396 v^{8} \\
& -4066106645832 v^{26}-85811470969983 v^{16}-3412629704 v^{38} \\
& +59293520 v^{42}-659156837224 v^{30}-487546443252 v^{4}+3762312617719 v^{24} \\
& +38057905046304 v^{12}-2478573368172 v^{6}-7934890634208 v^{14} \\
& \left.+21122195260320 v^{10}-527425668 v^{2}\right) u^{16}+\left(5849753404 v^{34}-2598156 v^{2}\right. \\
& +1562491386720 v^{12}-2182595382 v^{4}-275815463088 v^{26}-18470269672 v^{30} \\
& 10560 v^{46}-118426 v^{44}-38293932600 v^{6}+833720325372 v^{24}+1272984 v^{42} \\
& +297597214728 v^{10}-392074238358 v^{28}-2180590723872 v^{18}-617364 v^{40} \\
& -2590541276400 v^{16}+1655953978800 v^{22}-80969064 v^{38}+554586385176 v^{14} \\
& \left.-870137732 v^{36}-92837286864 v^{8}-+44924792088 v^{32}+638073143754 v^{20}\right) u^{8} \\
& +\left(193630617296952 v^{16}-112694973711156 v^{12}+1832097764 v^{40}+873477 v^{46}\right. \\
& +639181712840 v^{32}-4253381815617 v^{6}+330211819068 v^{34}-517405899879 v^{14} \\
& -15661888572462 v^{10}-15255667669 v^{38}-30543436 v^{44}-83914926220730 v^{22} \\
& +45403410492 v^{2}+25510822958500 v^{26}-3777649924752 v^{4}+184473146 v^{42} \\
& -134940248337048 v^{20}-45154052504 v^{36}+24868553257836 v^{8} \\
& -6309227266496 v^{28}+33996057356464 v^{24}+83226321847256 v^{18}
\end{aligned}
$$




$$
\begin{aligned}
& \left.-3582730793678 v^{30}\right) u^{28}-v^{24}-66 v^{28}+12 v^{26}+1884 v^{30}+109329 v^{32}+12 v^{46} \\
& -66 v^{44}+844388 v^{36}+109329 v^{40}+1884 v^{42}+824472 v^{38}+824472 v^{34}-v^{48} \\
& +\left(396 v^{46}+3752892 v^{6}-109516212 v^{8}+80572480 v^{36}-6465174408 v^{10}\right. \\
& -392747839 v^{34}-66585987564 v^{24}+102271662702 v^{18}-48413750220 v^{12} \\
& +70095543804 v^{16}-708588 v^{4}-83988 v^{42}-34071600888 v^{14}+59049 v^{2} \\
& +631860748 v^{30}-1095864 v^{38}-3414039948 v^{32}-81496125348 v^{22}+6336 v^{44} \\
& \left.-1558480 v^{40}+19467071940 v^{26}+19796882652 v^{20}+28507213020 v^{28}\right) u^{4}=0 .
\end{aligned}
$$

Proof. Using the equations (2.1) and (2.5), we arrive at the equation (3.5).

\section{REFERENCES}

[1] G. E. Andrews, An introduction to Ramanujan,s "Lost" notebook. Am. Math. Mon. 86, 89-108 (1976).

[2] B. C. Berndt, Ramanujan's Notebooks, Part III, Springer-Verlag, New York (1991).

[3] H.H. Chan, S.-S. Haung, On the Ramanujan-Göllnitz-Gordon continued fraction. Ramanujan J. 1, 75-90 (1997).

[4] H. Göllnitz, Partition mit Differnzebendingungen, J. Reine Angew. Math., 25, 154-190 (1967).

[5] B. Gordon, Some continued fractions of the Rogers-Ramanujan type, Duke Math. J., 32, 741-748 (1965).

[6] G. H. Hardy, Ramanujan, Chelsea, New York (1978).

[7] S. Ramanujan, Notebooks (2 volumes). Bombay. Tata Institute of Fundamental Research (1957).

[8] S. Ramanujan, The 'lost' notebook and other unpublished papers. New Delhi. Narosa (1988).

[9] L. J. Rogers, On a type of modular relation. Proc. Lond. Math. Soc., 19, 387-397 (1921).

[10] K. R. Vasuki, N. Bhaskar and G. Sharath, On a continued fraction of order six. Ann Univ Ferrara, Vol. 56, 77-89 (2010).

\section{Received: September, 2012}

